Check for updates

Cite this: RSC Adv., 2019, 9, 35532

\title{
Mono-acrylated isosorbide as a bio-based monomer for the improvement of thermal and mechanical properties of poly(methyl methacrylate)
}

\begin{abstract}
Dinghua Yu, iD *a Juan Zhao, ${ }^{b}$ Wenjuan Wang, ${ }^{b}$ Jingjie Qi ${ }^{\mathrm{a}}$ and $\mathrm{Yi} \mathrm{Hu}^{\mathrm{b}}$
Despite its optical clarity and good weatherability, poly(methyl methacrylate) (PMMA) cannot meet the needs of special occasions due to its deficient thermal and mechanical properties. To overcome these shortcomings, a type of novel bio-based monomer, mono-acrylated isosorbide, was used as a comonomer for the poly(methyl methacrylate) via a solution polymerization process. The chemical structure, the thermal and mechanical properties of the copolymerized PMMA were characterized. When the molar content of the mono-acrylated isosorbide was increased from $0 \%$ to $15 \%$, the glass transition temperature $T_{\mathrm{g}}$ of the copolymerized PMMA was increased from $151.2{ }^{\circ} \mathrm{C}$ to $172.5{ }^{\circ} \mathrm{C}$, and the initial decomposition temperature $\left(T_{5 \%}\right)$ was increased from $323.1{ }^{\circ} \mathrm{C}$ to $396.3{ }^{\circ} \mathrm{C}$. Moreover, the impact strength of copolymerized PMMA increased from $10.59 \mathrm{~kJ} \mathrm{~m}^{-2}$ to $17.19 \mathrm{~kJ} \mathrm{~m}^{-2}$ and the tensile strength improved from 84.02 $\mathrm{MPa}$ to $97.56 \mathrm{MPa}$ when the mono-acrylated isosorbide was incorporated with different contents. The incorporation of rigid and thermally stable isosorbide could contribute to the improved thermal and mechanical properties of PMMA, which would find important applications in the military and aeronautical materials under harsh service environments.
\end{abstract}

Received 18th September 2019 Accepted 15th October 2019

DOI: $10.1039 / \mathrm{c} 9 \mathrm{ra0} 07548 \mathrm{~h}$

rsc.li/rsc-advances inorganic nanofiller. In addition, a difficult blending process is required to acquire the even-distributed composite. Therefore, the copolymerization modification with a special comonomer could be a promising protocol to improve the thermal and mechanical performance without sacrificing the optical properties.

Being the focus of the polymer industry for the last decade, the utilization of bio-renewable feedstocks has produced many renewable building blocks with a special structure, which could be used to create novel polymer materials. For example, 2,5furandicarboxylic acid derived from carbohydrates can substitute petroleum-derived terephthalic acid and be used to synthesize bio-based plasticizers and polyesters with many outstanding properties. ${ }^{10,11}$ Bio-based long-chain diacid and diamine are also type of important monomers for bio-based polyamide, which has attracted broad interest due to numerous outstanding advantages, such as high strength, toughness, corrosion resistance and wears resistance. ${ }^{12-14}$ Therefore, bio-renewable feedstocks, including carbohydrates, triglycerides, and proteins, could bring new opportunities for the development of a novel functional polymer. ${ }^{15,16}$

1,4:3,6-Dianhydro-D-glucitol isosorbide can be produced from starch via chemical-biological processes, including enzymatic hydrolysis to glucose, chemical hydrogenation to sorbitol and chemical dehydration to isosorbide. Due to the typical advantages, such as rigidity, high thermal stability, renewability
${ }^{a}$ College of Biotechnology and Pharmaceutical Engineering, Nanjing Tech University, Nanjing 211816, China. E-mail: yudh@njtech.edu.cn; Fax: +86-25-58139389; Tel: $+86-25-58139386$

${ }^{b}$ College of Pharmaceutical Science, Nanjing Tech University, Nanjing 211816, China 
and non-toxicity, isosorbide has been used to prepare polyesters, polyethers, polyurethanes, polycarbonates, polyphosphonates, polytriazoles, and so on. ${ }^{17-24}$ For example, because of its non-toxicity, isosorbide has been used as a substitution for bisphenol $\mathrm{A}$ in polycarbonates and epoxy resins particularly for applications in contact with food. ${ }^{25}$ In other applications, isosorbide has been used as a comonomer to increase the glass transition temperature $\left(T_{\mathrm{g}}\right)$ and thermal stability of polyesters such as PET and PBS, ${ }^{23}$ which could broaden the high-temperature applications of polyesters such as the moulded parts or packaging materials.

Comparing with the direct use as a diol and cross-linking monomer, mono-vinyl isosorbide derivatives have been reported only in a few works of literature. ${ }^{26-32}$ These mono-vinyl isosorbide monomers are especially useful and could find important applications in the construction of polymers with designed architectures through chain-growth polymerizations. For example, Beghdadi et al. $^{31}$ reported that the substitution of the vinyl triazole moiety at either the endo- or exo-position had a significant effect on the glass transition temperature $T_{\mathrm{g}}$ and solubility of the polymer. Polymers prepared from a monomer with the vinyl triazole group at the exo-position were insoluble in water and possessed a $T_{\mathrm{g}}=118{ }^{\circ} \mathrm{C}$, whereas those prepared from a monomer with vinyl triazole at the endo-position were water-soluble and showed a $T_{\mathrm{g}}=71{ }^{\circ} \mathrm{C}$. However, there are few reports on PMMA modified with mono-vinyl isosorbide for improving the thermal and mechanical performance.

In this study, the special bio-based mono-vinyl monomer, mono-acrylated isosorbide (MAI), was synthesized through direct esterification of the isosorbide and acrylic acid with $p$ toluenesulfonic acid as the catalyst. Then, the copolymerized PMMA samples were prepared through solution polymerization with different molar contents of mono-acrylated isosorbide. The polymer structures were studied by Fourier transform infrared spectroscopy (FTIR) and ${ }^{1} \mathrm{H}$ nuclear magnetic resonance spectroscopy (NMR) techniques. The thermal and mechanical properties of the copolymerized PMMA materials were characterized by simultaneous thermal analysis, differential scanning calorimetry (DSC), dynamic mechanical analysis (DMA), impact strength and tensile strength experiments. The potential relationship between structure and performance was discussed.

\section{Experimental methods}

\section{Materials}

Isosorbide (98\%) was obtained from the Hongbaifeng Industry and trade Co., Ltd. (Jinan, China). Sodium chloride, sodium bicarbonate, acrylic acid (99\%, inhibited with 200 ppm monomethyl ether hydroquinone) and methyl methacrylate (98\%, inhibited with $250 \mathrm{ppm}$ mono-methyl ether hydroquinone) (MMA) were bought from Aladdin Co. Shanghai, China. $p$-Toluenesulfonic acid, chloroform, benzoyl peroxide, hydroquinone, methanol, acetone, toluene, and $\mathrm{CDCl}_{3}$ were acquired from Sinopharm Chemical Reagent Co., Ltd. Prior to the experiments, isosorbide was purified through ethyl acetate recrystallization. Methyl methacrylate was washed to remove the inhibitor by a $5 \%$ solution of sodium hydroxide. The oil phase liquid was separated and dried by anhydrous calcium chloride. Other reagents were used as received without further purification.

\section{Synthesis of mono-acrylated isosorbide}

As shown in Scheme 1, the direct esterification of isosorbide was performed using $p$-toluenesulfonic acid as a catalyst in a typical Dean-Stark apparatus. In order to minimize the polymerization of acrylic acid itself, hydroquinone was used as the inhibitor. In a typical experiment, a given amount of purified isosorbide and toluene (water carrier) were added into a round bottom flask. After isosorbide has melted after heating, $1.8 \%$ of $p$-toluenesulfonic acid (wt $\%$ based on total reactants) was added into the flask. Then, the acrylic acid containing $0.1 \%$ hydroquinone (wt\%) was added dropwise into the flask. In order to minimize the di-acrylated isosorbide formation, the molar ratio of the acrylic acid to isosorbide was fixed at $1: 1$. The reaction proceeded at $100^{\circ} \mathrm{C}$ for $4 \mathrm{~h}$. After cooling to room temperature, the crude products were washed using a saturated solution of $\mathrm{NaHCO}_{3}$ to remove unreacted acrylic acid, isosorbide, hydroquinone and $p$-toluenesulfonic acid, and the oil phase was further washed by saturated $\mathrm{NaCl}$ solution and dried over anhydrous $\mathrm{Na}_{2} \mathrm{SO}_{4}$. The crude product was further purified via flash column chromatography (eluent: methanol/acetone $=$ $3: 1, \mathrm{v} / \mathrm{v}$ ). The typically isolated yield based on isosorbide was $82.5-85.6 \%$.

\section{Free radical polymerization of MAI and MMA}

As shown in Scheme 2, mono-acrylated isosorbide was used as a comonomer for copolymerization with the purified MMA. During the solution polymerization, chloroform was used as the solvent. In a typical experiment, 0.1 mol purified methyl methacrylate and a given amount of mono-acrylated isosorbide (mol\%, based on MMA) was dissolved into $50 \mathrm{~mL}$ chloroform, and the solution was transferred into a round bottom flask. After the reactants solution was heated to $70{ }^{\circ} \mathrm{C}, 1 \%$ benzoyl peroxide initiator ( $\mathrm{wt} \%$ based on total reactants) of chloroform solution was added slowly. Then, the reactant mixtures were heated to $80{ }^{\circ} \mathrm{C}$ and remained for $5 \mathrm{~h}$. The un-reacted monomers and chloroform in the solution were rotary evaporated under vacuum. The acquired solid products were rinsed by acetone and methanol for three times and were dried at $40{ }^{\circ} \mathrm{C}$ under vacuum. Typically the isolated yield of the polymer based on the total monomer weight is $85.8-89.3 \%$. Similar to the parent PMMA, copolymerized PMMA samples with monoacrylated isosorbide show the $90-92 \%$ transmittance in the visible region $(400-700 \mathrm{~nm})$.

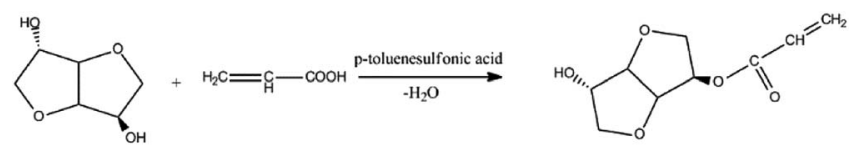

Scheme 1 Synthesis of mono-acrylated isosorbide. 
n
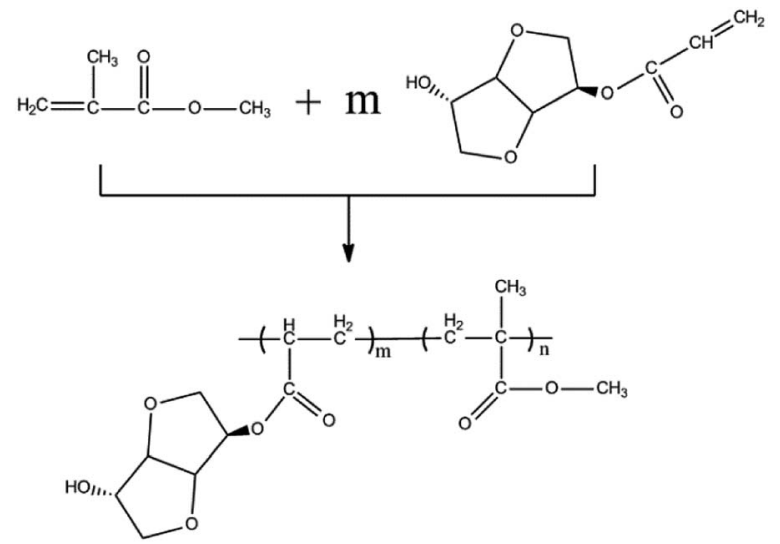

Scheme 2 Free radical copolymerization of methyl methacrylate and mono-acrylated isosorbide.

\section{Properties characterization}

Structure characterization. The molecular weight and polydispersity index (PDI) of copolymerized PMMA were determined via gel permeation chromatography (GPC), which was performed on Wyatt system equipped with an SSI 1500 pump and a Waters Styragel HR $2.5 \mu \mathrm{m}, 300 \mathrm{~mm} \times 7.8 \mathrm{~mm}$ column. Chloroform $\left(1.0 \mathrm{~mL} \mathrm{~min}{ }^{-1}\right)$ was used as the eluent at $30{ }^{\circ} \mathrm{C}$ using commercial polystyrene standards for calibration.

${ }^{1} \mathrm{H}$ NMR $(600 \mathrm{MHz})$ spectra were recorded on a Bruker Avance-III600 spectrometer in $\mathrm{CDCl}_{3}$ with tetramethylsilane (TMS) as the internal reference.

FTIR spectra were recorded on an Avatar 360 FTIR spectrometer from Nicolet with DTGS $\mathrm{KBr}$ as the detector. The sample was mixed together with $\mathrm{KBr}$ powder and tableted into a thin round piece. The piece was then scanned in the range of $4000-400 \mathrm{~cm}^{-1}$.

Thermal properties. Differential scanning calorimetry was performed using a dynamic scanning calorimeter (PerkinElmer, DSC/DSC7). The material was cooled down from ambient to $-30{ }^{\circ} \mathrm{C}$ with a ramp of $-5{ }^{\circ} \mathrm{C} \mathrm{min}^{-1}$ and maintained at $-30{ }^{\circ} \mathrm{C}$ for $30 \mathrm{~min}$ to ensure its thermal equilibrium. Then, the analysis was registered under air at a heating rate of $5{ }^{\circ} \mathrm{C} \mathrm{min}^{-1}$. The glass transition temperature $\left(T_{\mathrm{g}}\right)$ of these copolymerized PMMA samples was obtained from the peak of the heat flow curves.

The simultaneous thermal analysis was performed using a thermogravimetric analyzer (TGA) from Netzsch Instruments (NETZSCH, 409PC). Samples of 5-10 mg were placed in a platinum pan and heated from $20{ }^{\circ} \mathrm{C}$ to $800{ }^{\circ} \mathrm{C}$ at a $10{ }^{\circ} \mathrm{C} \mathrm{min}{ }^{-1}$ ramp under an inert $\mathrm{N}_{2}$ atmosphere.

The dynamic mechanical analysis was evaluated using a dynamic mechanical analyzer (TA Instruments, DMA Q800). The rectangular specimen $(20.0 \mathrm{~mm}(L) \times 5.0 \mathrm{~mm}(W) \times$ $0.1 \mathrm{~mm}(T)$ ) was made by casting the polymers in a mould. The DMA spectra were recorded in the temperature range of 30$300{ }^{\circ} \mathrm{C}$ at a $5{ }^{\circ} \mathrm{C} \mathrm{min}^{-1} \mathrm{ramp}$. The mechanical loss factor $(\tan \delta)$ has been recorded as a function of temperature. All the measurements were performed at a frequency of $1 \mathrm{~Hz}$. The glass transition temperature $\left(T_{\mathrm{g}}\right)$ of this modified PMMA has been acquired from the peak of the loss factor curves.
Mechanical properties. The Charpy impact strength was evaluated using an impact test machine (XCJ-4, Chengde, China) according to ISO 179-1-2001. The experimental impact strength was $1 \mathrm{~J}$, and the specimens span was $40 \mathrm{~mm}$. Each sample was determined repeatedly for five times, and the average impact strength value was the final result.

The tensile strength was determined on the universal mechanical tester (Instron Co. Shimadzu GE-I). The standard dumbbell-shaped specimens were prepared via casting methods. The experimental speed was $5 \mathrm{~mm} \min ^{-1}$. Each sample was determined repeatedly for five times to obtain the reliable average value.

\section{Results and discussion}

\section{Structural analysis of mono-acrylated isosorbide}

The structure of mono-acrylated isosorbide was characterized via FTIR and ${ }^{1} \mathrm{H}$ NMR, and the corresponding results are shown in Fig. 1. In order to compare the characteristic absorption, diacrylated isosorbide (DAI) is also shown in Fig. 1(A). As shown in Fig. 1(A), the characteristic peak at $1730 \mathrm{~cm}^{-1}$ could be ascribed to the stretching vibration of $-\mathrm{C}=\mathrm{O}$ bond, and the absorption at $1270 \mathrm{~cm}^{-1}$ and $1180 \mathrm{~cm}^{-1}$ could be ascribed to the stretching vibration of -C-O-C- bond, which indicates that ester bond was formed. The $\mathrm{C}=\mathrm{C}$ bond stretching vibration of the acrylate groups was observed at $1635 \mathrm{~cm}^{-1}$, and the absorption at $3080 \mathrm{~cm}^{-1}$ could be induced by the $-\mathrm{C}-\mathrm{H}$ stretching vibration of

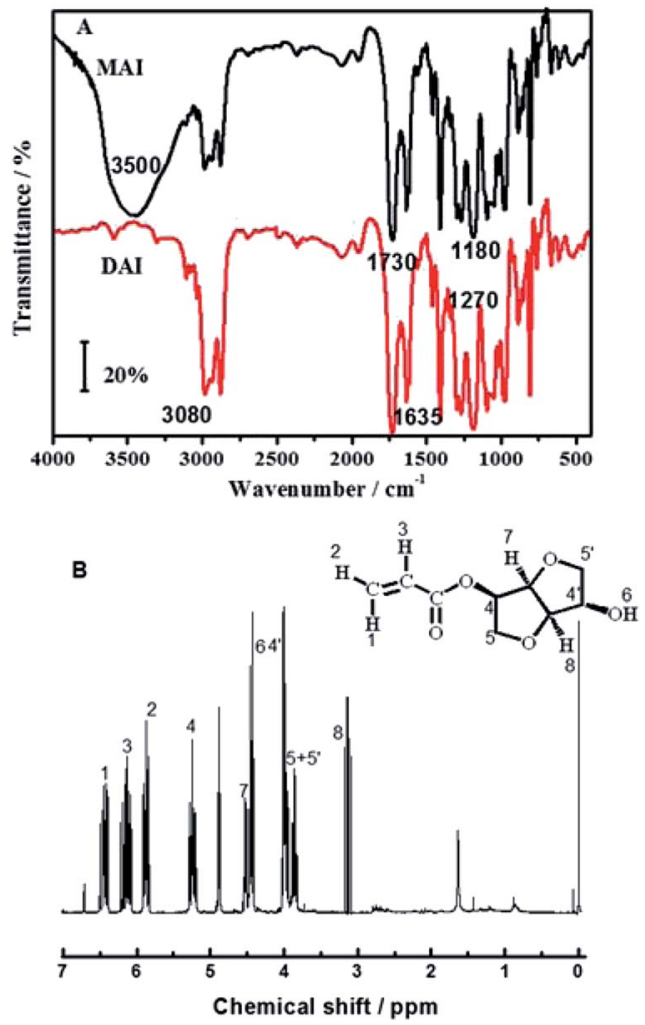

Fig. 1 FTIR and ${ }^{1} \mathrm{H}$ NMR spectra of mono-acrylated isosorbide. (A) FTIR; (B) ${ }^{1} \mathrm{H}$ NMR. 
$-\mathrm{C}=\mathrm{C}-\mathrm{H}$ groups, which demonstrates that an acrylate group has been introduced into the products. Comparing with DAI monomer, the MAI monomer showed an obvious absorption peak at $3500 \mathrm{~cm}^{-1}$, which could be ascribed to the $-\mathrm{O}-\mathrm{H}$ stretching vibration of isosorbide.

The ${ }^{1} \mathrm{H}$ NMR results shown in Fig. 1(B) demonstrate the structure of mono-acrylated isosorbide. The chemical shift at 6.40-6.43 ppm, 5.77-5.81 ppm, and 6.01-6.06 ppm could be ascribed to the proton 1, 2 and 3 of the vinyl group. The chemical shift at 5.43-5.48 ppm, 4.29-4.34 ppm, 4.17-4.19 ppm, 3.39-3.52 ppm and 4.79-4.81 ppm could be induced by the protons located at the isosorbide rings. The chemical shift at 4.7-4.73 ppm could be ascribed to the proton of free hydroxyl from the mono-acrylated isosorbide. These results indicate that mono-acrylated isosorbide was synthesized successfully.

\section{Polymer structural analysis}

In order to demonstrate the incorporation of mono-acrylated isosorbide, FTIR and ${ }^{1} \mathrm{H}$ NMR were used to study the structure of polymers. As shown in Fig. 2, the strong absorption at $1735 \mathrm{~cm}^{-1}$ could be ascribed to the stretching vibration of $-\mathrm{C}=$ $O$ bonds, and the absorption at $1150 \mathrm{~cm}^{-1}$ could be ascribed to the stretching vibration of $-\mathrm{C}-\mathrm{O}-\mathrm{C}$ bonds, which indicates that the ester bonds exist in the polymer. Two samples did not show absorption at $1635 \mathrm{~cm}^{-1}$, which is the typical absorption of $-\mathrm{C}=$ $\mathrm{C}$, indicating that the acrylate monomers have been polymerized totally. The absorption at $2990 \mathrm{~cm}^{-1}$ could be ascribed to saturated $\mathrm{C}-\mathrm{H}$ stretching vibration, and the absorption at $1470 \mathrm{~cm}^{-1}$ and $1390 \mathrm{~cm}^{-1}$ could be ascribed to the deformation vibration of $-\mathrm{CH}_{2}-\mathrm{CH}_{2}-$ and $-\mathrm{CH}_{3}$. It is a remarkable fact that there was a new medium absorption peak at $3630 \mathrm{~cm}^{-1}$ for PMMA-8\%MAI sample, compared with the parent PMMA. This absorption peak could be ascribed to the stretching vibration of the hydroxyl group located in the MAI monomer, which demonstrates that the MAI monomer was introduced into the skeleton of PMMA.

Mono-acrylated isosorbide could copolymerize with other vinyl monomers through free radical polymerization, but the rigid block of isosorbide could influence the polymerization

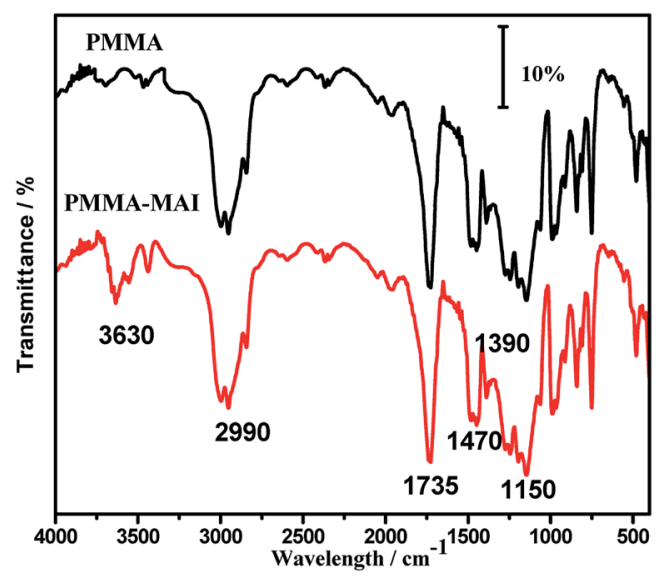

Fig. 2 FTIR spectra of parent PMMA and PMMA-8\%MAI. velocity and the final polymer molecular weights. Therefore, the effect of the ratio of MAI to MMA on molecular weight and PDI has been studied by the GPC method and the corresponding results have been listed in Table 1 . Comparing with the parent PMMA, the copolymerized PMMA samples with mono-acrylated isosorbide showed lower molecular weights and higher PDI values. On the other hand, with the increase in the monoacrylated isosorbide content, the copolymerized samples showed a decrease in the molecular weight and increase in the PDI value, which indicates that MAI has lower reactivity than MMA.

${ }^{1} \mathrm{H}$ NMR technique was used to study the structure of the parent and copolymerized PMMA, and the corresponding results are shown in Fig. 3. From Fig. 3(A), the chemical shift at 0.80-0.89 ppm and 0.92-1.01 ppm could be ascribed to the proton 1 and 2 of the methylene group in the parent PMMA. The broad chemical shift at 1.8-2.0 ppm could be ascribed to the proton of the methyl group. The strong chemical shift at 3.56$3.60 \mathrm{ppm}$ could be induced by the protons of methyl linked to the ester bond. In comparison, Fig. 3(B) shows more complicated chemical shift peaks. The chemical shift at 4.43-4.45 ppm could be ascribed to the proton 1 of the isosorbide hydroxyl group. The chemical shift at 3.84-3.86 ppm and 3.98-4.00 ppm could be ascribed to the proton 2 and 5 of the isosorbide ring. The chemical shift at 4.51-4.53 ppm could be ascribed to the proton 3 of the methylene group in isosorbide. These results demonstrate that the copolymer of MAI and MMA was synthesized successfully. According to the qualitative calculation, the actual content of mono-acrylated isosorbide is $7.12 \%$ in the PMMA-8\%MAI samples, lower than the theoretical value, which could be induced by the slower polymerization rate of the mono-acrylated isosorbide.

\section{Thermal properties}

Fig. 4 shows the TG and the corresponding DTG curves of the parent and copolymerized PMMA samples. The weight loss value of $5 \%$ was chosen as the reasonable criterion to evaluate the onset of the thermal degradation of each sample, and the results are listed in Table 2.

It was observed from the TG curves in Fig. 4(A) that all of the PMMA samples were thermally stable in the $\mathrm{N}_{2}$ atmosphere below $250{ }^{\circ} \mathrm{C}$ and were seen to undergo a one-stage thermal degradation process above this temperature. According to Czech's report, ${ }^{33}$ the PMMA could degrade via a homolytic

Table 1 Experimental molecular weight of PMMA-MAI polymer samples

\begin{tabular}{lllll}
\hline Samples & $n($ MAI $): n($ MMA $)$ & $M_{\mathrm{w}}$ & $M_{\mathrm{n}}$ & PDI \\
\hline PMMA & 0 & 78326 & 73892 & 1.02 \\
PMMA-2\%MAI & $2: 100$ & 74213 & 55799 & 1.33 \\
PMMA-5\%MAI & $5: 100$ & 69856 & 54152 & 1.29 \\
PMMA-8\%MAI & $8: 100$ & 70684 & 51974 & 1.36 \\
PMMA-10\%MAI & $10: 100$ & 76358 & 55736 & 1.37 \\
PMMA-15\%MAI & $15: 100$ & 71426 & 50657 & 1.41
\end{tabular}



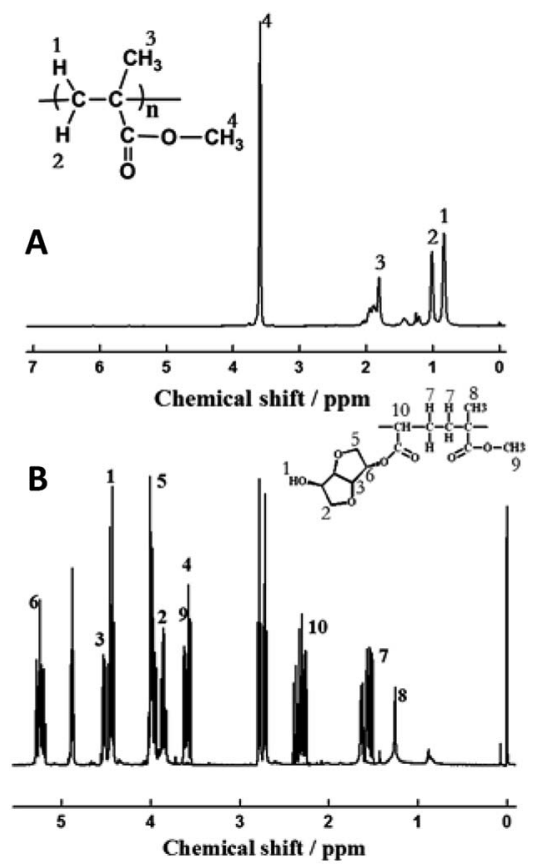

Fig. $3{ }^{1} \mathrm{H}$ NMR spectra of samples. (A) Parent PMMA; (B) PMMA-8\% MAI.

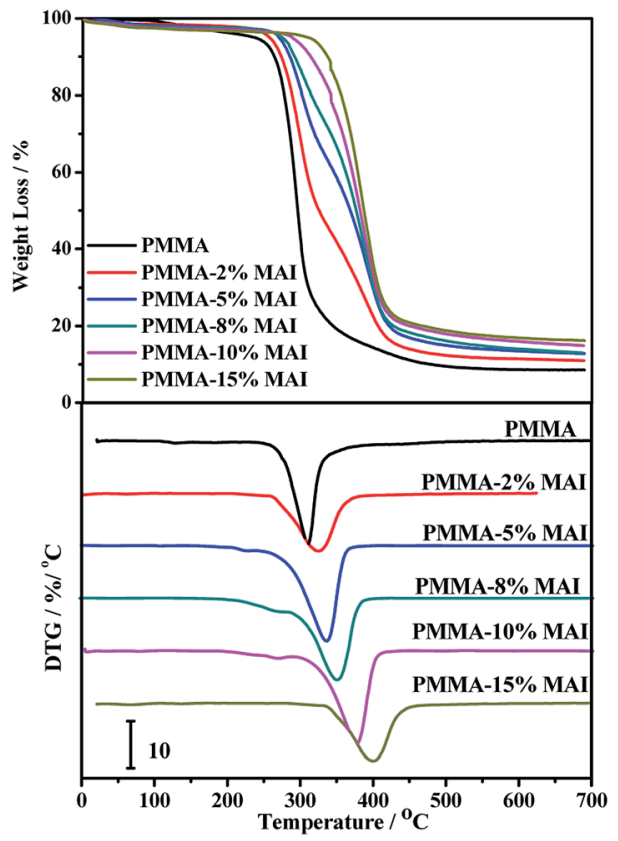

Fig. 4 Thermal analysis profiles of parent PMMA and PMMA-MAI.

polymer chain cleavage, followed by monomer unzipping. Comparing with the sharp downstream decomposition curve of the parent PMMA sample, the copolymerized PMMA samples showed gentle downstream decomposition curves and the typical narrower decomposition temperature ranges, which indicate that the copolymerized PMMA samples have undergone complicated decomposition via different decomposition
Table 2 Glass transition temperature and thermal stability of parent PMMA and PMMA-MAI samples

\begin{tabular}{lllll}
\hline PMMA-MAI polymers & $\begin{array}{l}T_{\mathrm{g}}\left({ }^{\circ} \mathrm{C}\right) \\
(\mathrm{DMA})\end{array}$ & $\begin{array}{l}T_{\mathrm{g}}\left({ }^{\circ} \mathrm{C}\right) \\
(\mathrm{DSC})\end{array}$ & $T_{5 \%}\left({ }^{\circ} \mathrm{C}\right)$ & $T_{\max }\left({ }^{\circ} \mathrm{C}\right)$ \\
\hline PMMA & 151.2 & 152.0 & 323.1 & 370.2 \\
PMMA-2\%MAI & 158.4 & 158.6 & 341.9 & 390.5 \\
PMMA-5\%MAI & 163.6 & 164.2 & 360.0 & 400.7 \\
PMMA-8\%MAI & 164.7 & 165.1 & 378.5 & 421.4 \\
PMMA-10\%MAI & 167.3 & 168.0 & 388.1 & 429.9 \\
PMMA-15\%MAI & 172.5 & 173.0 & 396.3 & 445.1
\end{tabular}

mechanisms due to the incorporation of isosorbide structure blocks.

From Fig. 4 and the data in Table 2, $T_{5 \%}$ and $T_{\max }$ for the parent PMMA was about $323^{\circ} \mathrm{C}$ and $370.2{ }^{\circ} \mathrm{C}$ under the classical temperature ramp $\beta$ of $10{ }^{\circ} \mathrm{C} \mathrm{min}^{-1}$, respectively. For general commercial resins, $T_{\max }$ is higher than $400{ }^{\circ} \mathrm{C}$ to meet polymer processing requirements. ${ }^{34,35}$ With the increase in the amount of mono-acrylated isosorbide from $2 \%$ to $5 \%, 8 \%, 10 \%$ and $15 \%$, the corresponding degradation temperature $T_{5 \%}$ increased from $342{ }^{\circ} \mathrm{C}$ to $360{ }^{\circ} \mathrm{C}, 379{ }^{\circ} \mathrm{C}, 388^{\circ} \mathrm{C}$ and $396{ }^{\circ} \mathrm{C}$. Moreover, the maximum degradation temperature $T_{\max }$ has increased from $370{ }^{\circ} \mathrm{C}$ to $391{ }^{\circ} \mathrm{C}, 401{ }^{\circ} \mathrm{C}, 421{ }^{\circ} \mathrm{C}, 430{ }^{\circ} \mathrm{C}$, and $445{ }^{\circ} \mathrm{C}$. These results indicate that the thermal stability of the copolymerized PMMA with the mono-acrylated isosorbide has obviously improved, which could be ascribed to the high thermal stability of the isosorbide. More significantly, these results demonstrate clearly that the copolymerization with mono-acrylated isosorbide could improve the thermal stability of the PMMA resins, meeting the thermal stability requirement of high-temperature commercial resins.

Fig. 5 shows the DSC curves of the parent and copolymerized PMMA samples, and the calculated glass transition temperature $\left(T_{\mathrm{g}}\right)$ are listed in Table 2. From Fig. 5, all of the samples showed a similar peak shape regardless of the MAI amount, and the tendency to shift to a higher temperature has been observed.

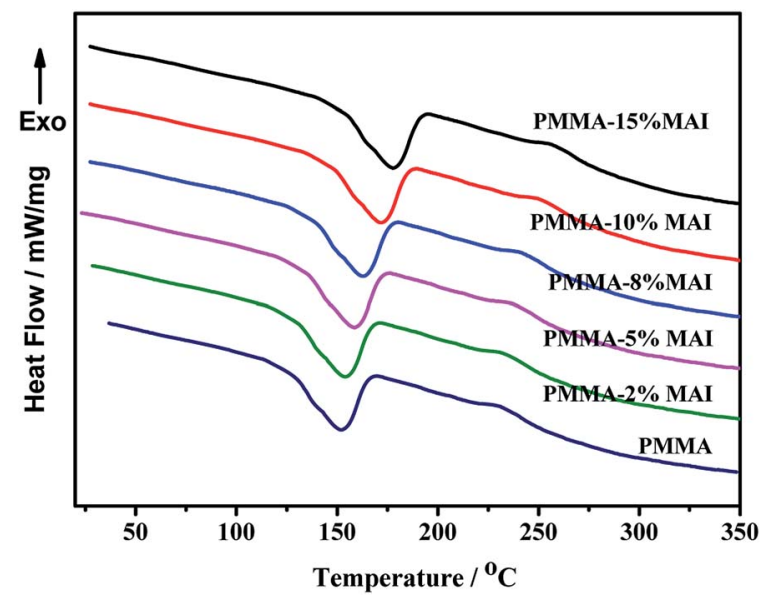

Fig. 5 DSC profiles of parent PMMA and PMMA-MAI. 
As shown in Table 2, the glass transition temperature $\left(T_{\mathrm{g}}\right)$ has increased from $152.0{ }^{\circ} \mathrm{C}$ to $158.6{ }^{\circ} \mathrm{C}, 164.2{ }^{\circ} \mathrm{C}, 165.1{ }^{\circ} \mathrm{C}$, $168.0{ }^{\circ} \mathrm{C}$ and $173.0{ }^{\circ} \mathrm{C}$ with the MAI increasing from 0 to $15 \%$.

The dynamic mechanical analysis was employed to investigate the variation of the $T_{\mathrm{g}}$ and mechanical properties of the parent PMMA and copolymerized PMMA as the temperature changes, and the results are shown in Fig. 6. As usual, the temperature corresponding to the tan $\delta$ peak maximum is taken as $T_{\mathrm{g}}$, and the results are listed in Table 2. From Table 2, the glass transition temperature $\left(T_{\mathrm{g}}\right)$ calculated from the DMA and DSC curves are very similar. The introduction of mono-acrylated isosorbide could enhance the intermolecular interactions of PMMA chains and increase the free volume of PMMA. Therefore, the incorporation of the mono-acrylated isosorbide decreased the mobility of the PMMA chains, which presented as the increase of glass transition temperature $T_{\mathrm{g}}$. According to Jannasch's viewpoint, ${ }^{36}$ the improved glass transition temperature $\left(T_{\mathrm{g}}\right)$ value for the polymers containing mono-vinyl isosorbide could originate from the combination of the rigid bicyclic structure and the hydrogen bond of the isosorbide monomers. As shown in Fig. 6, it is clear that each sample has only one $\tan \delta$ peak, indicating that the copolymerized PMMA is a thermodynamically compatible copolymer. Besides, the peak width of $\tan \delta$ could be related to the miscibility between the comonomers, and the narrow peak indicates good miscibility between different blocks of copolymers. From the DMA curves in Fig. 6, the $\tan \delta$ peak becomes gentler and wider with the increase in the MAI amount, indicating that mono-acrylated isosorbide with the PMMA chain has lower miscibility, which could be induced by the free hydroxyl group of isosorbide.

\section{Mechanical properties}

The mechanical properties of the parent and copolymerized PMMA are presented in Table 3. As shown in Table 3, the parent PMMA showed a tensile strength of $84.02 \mathrm{MPa}$. Interestingly, the tensile strength of the copolymerized PMMA-2\%MAI was 89.13 MPa, which increased by $6.08 \%$. With the increase in the mono-acrylated isosorbide from $5 \%$ to $8 \%$, the tensile strength of the copolymerized PMMA increased further to 93.19 $\mathrm{MPa}$ and

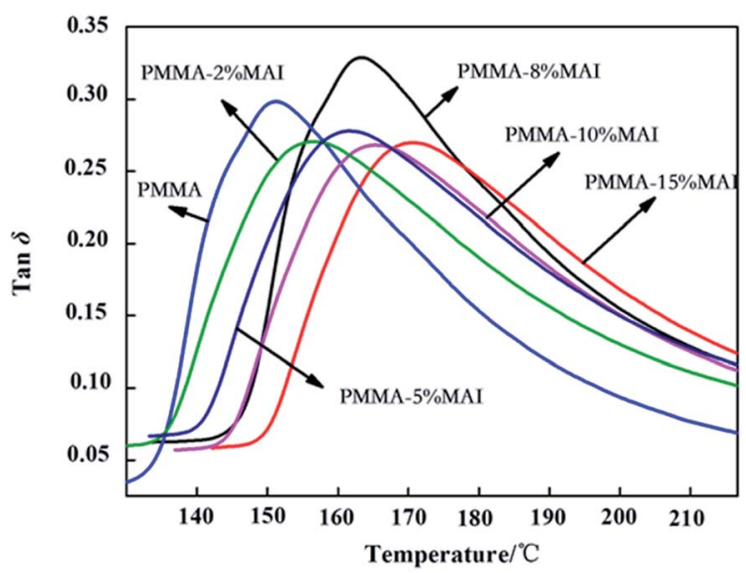

Fig. 6 DMA profiles of parent PMMA and PMMA-MAI samples.
Table 3 Mechanical properties of parent PMMA and PMMA-MAI samples

\begin{tabular}{lll}
\hline Samples & Impact strength/kJ m & Tensile strength/MPa \\
\hline PMMA & $10.59 \pm 0.85$ & $84.02 \pm 2.83$ \\
PMMA-2\%MAI & $13.24 \pm 0.6$ & $89.13 \pm 3.25$ \\
PMMA-5\%MAI & $15.08 \pm 0.78$ & $93.19 \pm 2.49$ \\
PMMA-8\%MAI & $16.51 \pm 0.89$ & $95.76 \pm 4.22$ \\
PMMA-10\%MAI & $16.97 \pm 1.21$ & $94.26 \pm 3.68$ \\
PMMA-15\%MAI & $17.19 \pm 0.93$ & $92.3 \pm 3.82$
\end{tabular}

95.76 MPa. According to Wissinger's standpoint, ${ }^{37}$ a higher tensile strength could originate from denser polymer chain packing and stronger intramolecular interactions. When the MAI monomer was copolymerized with MMA, the hydroxyl group of the isosorbide block enhanced further interaction among the polymer chains, which could contribute to the improved tensile strength. When the MAI content was improved further to $10 \%$ and $15 \%$, the tensile strength of the copolymerized PMMA decreased to 94.26 MPa and 92.3 MPa. The introduction of excessive MAI monomer to the PMMA chain could induce the highly branched PMMA chains, increased the distance between the polymer chains, and weakened the interactions between the chains, which could contribute to the decreased tensile strength.

From Table 3, the impact strength of the parent PMMA was observed as $10.59 \mathrm{~kJ} \mathrm{~m}^{-2}$, and the copolymerized PMMA-2\% MAI showed $13.24 \mathrm{~kJ} \mathrm{~m}^{-2}$, increased by $25.02 \%$. When the MAI amount was improved further from $5 \%$ to $8 \%, 10 \%$, and $15 \%$, the copolymerized PMMA samples showed an improved impact strength from $15.08 \mathrm{~kJ} \mathrm{~m}^{-2}$ to $16.51 \mathrm{~kJ} \mathrm{~m}^{-2}, 16.97 \mathrm{~kJ} \mathrm{~m}^{-2}$, and $17.19 \mathrm{~kJ} \mathrm{~m}^{-2}$, respectively. Comparing with the linear PMMA chain, the incorporation of MAI with the free hydroxyl group increased the intramolecular interactions via a hydrogen bond. These enhanced interactions could inhibit the chain cleavage, improve impact strength, and contribute to a better toughness, which are in agreement with the results reported by Tu et al. ${ }^{38} \mathrm{In}$ addition, the introduction of a fused five-member rings of isosorbide into the PMMA linear chains has increased the free volume of the polymer chains, which could further improve the PMMA toughness.

\section{Conclusion}

In summary, a novel bio-based mono-vinyl monomer was synthesized with isosorbide as the core scaffold. With the mono-acrylated isosorbide as a comonomer, the thermal and mechanical properties of PMMA could be improved obviously. The introduction of the isosorbide block as PMMA side chains could inhibit the polymer chain flexibility, and enhance the interactions among PMMA chains via a hydrogen bond, increasing the free volume of PMMA chains, which could contribute to the improved thermal and mechanical properties. Overall, the results of this study show that the mono-vinyl monomer of isosorbide can be used to improve the thermal and mechanical properties of PMMA, which could find 
important applications in the military and aeronautical materials under harsh service environments.

\section{Conflicts of interest}

There are no conflicts to declare.

\section{Acknowledgements}

The authors are grateful for the financial support by the National Natural Science Foundation of China (No. 21376120), the National Key Technology R\&D Program (No. 2012BAD32B08), and Six Talent Peaks Project in Jiangsu Province (No. 2018-GDZB-196).

\section{References}

1 F. Fenouillot, A. Rousseau, G. Colomines, R. Saint-Loup and J. P. Pascault, Prog. Polym. Sci., 2010, 35, 578-622.

2 D. A. Garlotta, J. Polym. Environ., 2001, 9, 63-84.

3 S. Gross, D. Camozzo, V. Di Noto, L. Armelao and E. TonDello, Eur. Polym. J., 2007, 43, 673-696.

4 S. C. Tjong, Mater. Sci. Eng., R, 2006, 53, 73-197.

5 Z. Spitalsky, D. Tasis, K. Paragelis and G. Costas, Prog. Polym. Sci., 2010, 5, 357-401.

6 M. T. Byrne and Y. K. Gun'ko, Adv. Mater., 2010, 22, 16721688.

7 K. Hayashida and Y. Matsuoka, Carbon, 2015, 85, 363-371.

8 S. L. Bee, M. A. A. Abdullah, M. Mamat, S. T. Bee, L. T. Sin, D. Hui and A. R. Rahmat, Composites, Part B, 2017, 110, 83-95.

9 D. B. Stojanović, L. Brajović, A. Orlović, D. Dramlić, V. Radmilović, P. S. Uskoković and R. Aleksić, Prog. Org. Coat., 2013, 76, 626-631.

10 A. H. Motagamwala, W. Won, C. Sener, D. M. Alonso, C. T. Maravelias and J. A. Dumesic, Sci. Adv., 2018, 4, eaap9722.

11 Q. Ouyang, J. Liu, C. Li, L. Zheng, Y. Xiao, S. Wu and B. Zhang, Polym. Chem., 2019, 10, 5594-5601.

12 L. Wang, X. Dong, P. Zhu, X. Zhang, X. Liu and D. Wang, Eur. Polym. J., 2017, 90, 171-182.

13 D. A. Ruehle, C. Perbix, M. Castaneda, J. R. Dorgan, V. Mittal, P. Halley and D. Martin, Polymer, 2013, 54, 6961-6970.

14 N. A. Jones, E. D. T. Atkins, M. J. Hill, S. J. Cooper and L. Franco, Macromolecules, 1997, 30, 3569-3578.

15 J. A. Galbis, M. G. Garcia-Martin, M. Violante and E. Galbis, Chem. Rev., 2016, 116, 1600-1636.
16 V. Sharma and P. P. Kundu, Prog. Polym. Sci., 2008, 32, 11991215.

17 S. Chatti, G. Schwarz and H. R. Kricheldorf, Macromolecules, 2006, 39, 9064-9070.

18 J. J. Gallagher, M. A. Hillmyer and T. M. Reineke, ACS Sustainable Chem. Eng., 2015, 3, 662-667.

19 M. Okada and K. Aoi, Curr. Trends Polym. Sci., 2002, 7, 57-70.

20 B. A. J. Noordover, R. Duchateau, R. van Benthem, W. Ming and C. E. Koning, Biomacromolecules, 2007, 8, 3860-3870.

21 L. Jasinska and C. E. Koning, J. Polym. Sci., Part A: Polym. Chem., 2010, 48, 2885-2895.

22 M. Beldi, R. Medimagh, S. Chatti, S. Marque, D. Prim, A. Loupy and F. Delolme, Eur. Polym. J., 2007, 43, 3415-3433.

23 R. T. Duan, Q. X. He, X. Dong, D. F. Li, X. L. Wang and Y. Z. Wang, ACS Sustainable Chem. Eng., 2016, 4, 350-362.

24 Y. B. Wang, J. B. Zhao, X. S. Li, T. Liu and D. H. Yu, Acta Polym. Sin., 2016, 40-45.

25 M. Rose and R. Palkovits, ChemSusChem, 2012, 5, 167-176. 26 L. Fertier, M. Ibert, C. Buffe, R. Saint-Loup, C. Joly-Duhamel, J. J. Robin and O. Giani, Prog. Org. Coat., 2016, 99, 393-399.

27 J. M. Sadler, A. P. T. Nguyen, F. R. Toulan, J. P. Szabo, G. R. Palmese, C. Scheck, S. Lutgen and J. J. La Scala, J. Mater. Chem. A, 2013, 1, 12579-12586.

28 J. J. Gallagher, M. A. Hillmyer and T. M. Reineke, ACS Sustainable Chem. Eng., 2016, 4, 3379-3387.

29 J. J. Gallagher, M. A. Hillmyer and T. M. Reineke, ACS Sustainable Chem. Eng., 2015, 3, 662-667.

30 Y. Mansoori, S. Hemmati, P. Eghbali, M. R. Zamanloo and G. Imanzadeh, Polym. Int., 2013, 62, 280-288.

31 S. Beghdadi, I. A. Miladi, H. Ben Romdhane, J. Bernard and E. Drockenmuller, Biomacromolecules, 2012, 13, 4138-4145.

32 Y. Mansoori, S. Hemmati, P. Eghbali, M. R. Zamanloo and G. Imanzadeh, Polym. Int., 2013, 62, 280-288.

33 Z. Czech, K. Agnieszka, P. Raganska and A. Antosik, J. Therm. Anal. Calorim., 2015, 119, 1157-1161.

34 J. J. La Scala, J. M. Sands, J. A. Orlicki, E. J. Robinette and G. R. Palmese, Polymer, 2004, 45, 7729-7737.

35 A. Chatterjee, J. Appl. Polym. Sci., 2009, 114, 1417-1425.

36 L. Matt, J. Parve, O. Parve, T. Pehk, T. H. Pham, I. Liblikas, L. Vares and P. Jannasch, ACS Sustainable Chem. Eng., 2018, 6(12), 17382-17390.

37 P. A. Wilbon, J. L. Swartz, N. R. Meltzer, J. P. Brutman, M. A. Hillmyer and J. E. Wissinger, ACS Sustainable Chem. Eng., 2017, 5(10), 9185-9190.

38 W. Wang, F. Wu, H. Lu, X. Li, X. Yang and Y. Tu, ACS Appl. Polym. Mater., 2019, 1, 2313-2321. 